\title{
| Preparação da mulher para a realização do exame de Papanicolaou na perspectiva da qualidade
}

\author{
Woman's preparation for the Papanicolaou's exam in the perspective of the quality
}

Preparación de la mujer para la realización del examen de Papanicolaou en la perspectiva de la calidad

\section{Kylvia Gradênia Torres Eduardo', Camila Félix Américo², Escolástica Rejane Moura Ferreira ${ }^{3}$, Ana Karina Bezerra Pinheiro $^{4}$, Lorena Barbosa Ximenes ${ }^{5}$}

\section{RESUMO}

Objetivo: avaliar a preparação da mulher para a realização do exame de Papanicolaou. Métodos: os dados foram coletados por meio da observação direta, seguindo o que preconiza o Instrumento de Melhoria de Desempenho (IMD), durante o mês de setembro de 2005. Participaram os sete enfermeiros do Programa Saúde da Família de um município cearense, os quais tiveram suas práticas observadas nos respectivos sítios de atuação (Unidade Básica de Saúde). Cada enfermeiro foi acompanhado em três exames, totalizando 21 observações. Resultados: dentre as 21 observações realizadas, em nenhuma, os enfermeiros endagaram se a usuária havia esvaziado a bexiga; em 8 $(38,1 \%)$ estas profissionais explicaram sobre o exame; e, em 18 (85,7\%, respectivamente orientaram para a troca de roupa em local privativo e cobririam a usuária de forma adequada durante o exame. Conclusões: a preparação das mulheres mostrou-se insatisfatória, pois os quatro itens preconizados no IMD só foram cumpridos em 52,4\% das vezes. Transformar esta realidade exige mudanças de atitude humana, área da competência técnica de maior desafio no contexto do serviço público.

Descritores: Esfregaço vaginal; Qualidade da assistência à saúde; Cuidados de enfermagem

\begin{abstract}
Objective: To evaluate nurses' competence in preparing women for Papanicolaou's smear according to the Performance Improvement Instrument. Methods: Data were collected through direct observation of the performance of 7 nurses from the Family's Health Program in Ceará, during the month of September, 2005. Each nurse was observed on three occasions. Results: The majority of nurses (85.7\%) provided privacy for the woman to change her clothes and maintained her privacy during the procedure by covering them properly. However, less than a half of the nurses $(38.2 \%)$ explained the procedure to the client, and none of the nurses inquired if the woman had emptied her bladder. Conclusion: the nurse competence for preparing women for Papanicolaou's smear was unsatisfactory because the four items of the Performance Improvement Instrument were performed only $52.4 \%$ of the time. Promoting changes in attitudes toward competence and adequate performance is challenging public health service.
\end{abstract}

Keywords: Vaginal smears; Quality of health care; Nursing care

\section{RESUMEN}

Objetivo: evaluar la preparación de la mujer para la realización del examen de Papanicolaou. Métodos: los datos fueron recolectados por medio de la observación directa, siguiendo lo que preconiza el Instrumento de Mejoría del Desempeño (IMD), durante el mes de setiembre del 2005. Participaron los siete enfermeros del Programa Salud de la Familia de un municipio cearense, los fueron cuales tuvieron observados durante la realización de sus prácticas clínicas en los respectivos locales de actuación (Unidad Básica de Salud). Cada enfermero fue acompañado en tres exámenes, con un total de 21 observaciones. Resultados: de las 21 observaciones realizadas, en ninguna de ellas los enfermeros preguntaron si la usuaria habia orinado; en $8(38,1 \%)$ estas profisionales dieron explicaciones sobre el examen y en $18(85,7 \%)$, respectivamente, orientaron sobre el local privativo para cambiarse y cubrieron la usuaria de forma adecuada durante el examen. Conclusiones: la preparación de las mujeres se mostró insatisfactoria, pues los cuatro itens recomendados en el IMD sólo fueron cumplidos en el 52,4\% de las veces. Transformar esta realidad exige cambios de actitud humana, área de competencia técnica de mayor desafío en el contexto del servicio público. Descriptores: Frotis vaginal; Calidad de la atención de salud; Atención de enfermería

${ }^{1}$ Pós-graduanda em Enfermagem na Promoção da Saúde pela Universidade Federal do Ceará - UFC - Fortaleza (CE), Brasil.

2 Acadêmica de Enfermagem; Bolsista do Programa de Educação Tutorial- PET-Enfermagem-Universidade Federal do Ceará - UFC - Fortaleza (CE), Brasil.

${ }^{3}$ Doutora em Enfermagem; Professora Adjunta I da Universidade Federal do Ceará - UFC - Fortaleza (CE), Brasil.

${ }^{4}$ Doutora em Enfermagem; Professora Adjunta II da Universidade Federal do Ceará - UFC. Co-Tutora do Programa de Educação Tutorial - PETEnfermagem - Fortaleza (CE), Brasil.

${ }^{5}$ Doutora em Enfermagem; Professora Adjunta III da Universidade Federal do Ceará - UFC. Tutora do Programa de Educação Tutorial - PET-Enfermagem - Fortaleza (CE), Brasil. 


\section{INTRODUÇÃO}

A qualidade é, com freqüência, o aspecto central a ser considerado para avaliação em saúde e está estreitamente relacionada com efetividade, eficácia, adequação e conhecimento técnico-científico ${ }^{(1)}$.

A busca por serviços de qualidade tem se tornado, cada vez mais, a meta dos serviços de saúde. O conceito de qualidade abrange satisfação do cliente (principal alvo dos cuidados de enfermagem) e dos profissionais, maior segurança e eficácia das ações prestadas e garantia de acesso a esses serviços ${ }^{(2)}$.

Esta busca parte principalmente de um questionamento interno do indivíduo que poderá vir, tanto daquele que fornece quanto do que recebe uma ação ou serviço. Portanto, as características de qualidade de um serviço ou produto devem ser definidas, levando-se também em consideração as necessidades do cliente ${ }^{(3)}$.

Alguns autores afirmam que o exame de prevenção do câncer de colo uterino é considerado, por muitas mulheres, um procedimento invasivo, que gera medo, vergonha, ansiedade, desconforto, repulsa à própria genitália e prolongados adiamentos na procura do serviço de saúde ${ }^{(4-5)}$.

Neste contexto, verifica-se que o exame de Papanicolaou exige de quem o realiza postura técnica e ética, no sentido de preservar a privacidade da cliente, posicioná-la de maneira confortável, compreendendo e participando do procedimento ao qual está sendo submetida.

Alguns elementos descritos como fundamentais para a qualidade da assistência em planejamento familiar, por exemplo, podem ser observados também no atendimento de prevenção de câncer de colo uterino, incluindo-se: informação aos usuários; competência técnica dos provedores de serviços; relação interpessoal cliente/ provedor; continuidade e seguimento do cliente; rede apropriada de serviços; e livre escolha sobre o tratamento ou procedimento ${ }^{(6)}$.

Médicos e enfermeiros devidamente treinados estão aptos a realizar o exame de Papanicolaou, que é considerado o método de rastreamento universal para câncer de colo uterino ${ }^{(7)}$. No cotidiano das equipes do Programa de Saúde da Família (PSF), o enfermeiro está engajado em todas as atividades de prevenção do câncer cérvico-uterino, sendo relevante seu papel quando se enfatiza que o maior número de coletas citológicas, em nível da atenção básica, é realizado por enfermeiros ${ }^{(8)}$.

A partir do exposto, e tomando-se por base o pressuposto de que para se adotar um programa de saúde reprodutiva de qualidade em uma unidade básica, o primeiro passo é fazer um diagnóstico das necessidades e problemas em relação ao atendimento ${ }^{(9)}$, elaboraram-se os seguintes questionamentos: os enfermeiros estão preparando as mulheres adequadamente para a realização do exame de Papanicolaou? Seguem o que preconiza o Programa de Qualidade-PROQUALI da Secretaria da Saúde do Ceará?

Com o intuito de responder a tais indagações, realizouse o presente estudo com o objetivo de avaliar a preparação da mulher para a realização do exame de Papanicolaou.

\section{MÉTODOS}

Estudo descritivo-exploratório com abordagem quantitativa, possibilitando assegurar a objetividade e credibilidade dos achados, além da quantificação dos mesmos $^{(10)}$. Foi assim classificado por utilizar-se de técnica padronizada para coleta de dados, objetivando descrever e explorar uma determinada atuação prática ${ }^{(11)}$.

Foi realizado entre enfermeiros do PSF de um município do Ceará que realizavam atendimento de prevenção do câncer de colo uterino. O PSF neste município conta com sete equipes em todo o seu território, o que corresponde a sete enfermeiros inseridos no Programa, atuando na área de prevenção de câncer de colo uterino e, portanto, realizando a coleta de material para o exame de Papanicolaou.

Foram selecionados sete enfermeiros do PSF do município em estudo que realizavam atendimento de prevenção do câncer de colo uterino e aceitaram participar da pesquisa voluntariamente.

$\mathrm{O}$ instrumento de coleta de dados foi o Instrumento de Melhoria do Desempenho (IMD) do Projeto PROQUALI, aplicado através da observação sistemática e direta dos enfermeiros, nos seus respectivos locais de trabalho (Unidades Básicas de Saúde).

O PROQUALI representa a ferramenta de acompanhamento e capacitação das equipes do PSF do Estado do Ceará. Foi institucionalizado na SESA-CE (Secretaria de Saúde do Ceará) como a "Metodologia de Melhoria do Desempenho" na atenção primária, tendo surgido de uma parceria entre o Governo do Ceará e algumas instituições internacionais (Fundo de População das Nações Unidas -FNUAP, Johns Hopkins Program for International Training in Reproductive Health JHPIEGO, Johns Hopkins University/Center for Communication Programs-JHU/CCP, Management Sciences for Health -MSH e Pathfinder do Brasil). É constituído por Instrumentos de Melhoria de Desempenho (IMD), os quais consistem em protocolos da estrutura do serviço e das ações prestadas pela equipe de PSF, tendo como pressupostos básicos a auto-avaliação e a autogestão(12).

O IMD dirigido para a prevenção do câncer cervical é composto por 20 procedimentos que descrevem o atendimento, passo a passo.

Como parâmetro de avaliação, o PROQUALI sugere que quando o procedimento for realizado adequadamente 
o mesmo seja categorizado como SIM, quando não for realizado ou for realizado inadequadamente seja categorizado como NÃO e quando a situação específica de um atendimento não se fizer necessária a realização de um determinado passo, este seja categorizado como "Não se Aplica"(12).

O instrumento apresenta a prevenção do câncer de colo uterino em etapas: anamnese, preparação da cliente para o exame, técnica de coleta propriamente dita e intervenções/encaminhamentos. O presente estudo delimitou como objeto de investigação os procedimentos referentes a preparação da cliente, os quais incluem: verifica se a usuária esvaziou a bexiga; explica a usuária o que vai ser feito; orienta a usuária a trocar de roupa atrás da cortina ou biombo, ou no banheiro do consultório, oferecendolhe bata, se preciso, e cobre a usuária adequadamente com lençol durante exame físico e/ou ginecológico, se preciso.

Para avaliação global do desempenho das participantes foi estabelecida a seguinte escala: desempenho satisfatório para os procedimentos que tiveram freqüência relativa de sim e/ou não se aplica acima de $90 \%$; desempenho intermediário para freqüência relativa entre $70 \%$ e $90 \%$ e desempenho insatisfatório para freqüência relativa abaixo de $70 \%$.

Os dados foram coletados no mês de setembro de 2005. Os enfermeiros foram contactados previamente, e a observação foi agendada, de acordo com o cronograma de cada um. Estes foram esclarecidos sobre a pesquisa e seus direitos enquanto participantes. Cada enfermeiro teve três atendimentos observados, totalizando 21 procedimentos.

De acordo com o PROQUALI, ao se utilizar o IMD para efeito de avaliação, cada procedimento deverá ser observado, no mínimo, em três situações, com a finalidade de se evitar falsas avaliações por situações "maquiadas" pelos participantes. Esse aspecto é corroborado por outros autores que orientam para a mesma estratégia, ressaltando o princípio da "reatividade", resposta do sujeito observado, que é capaz de alterar os resultados reais de um estudo ${ }^{(13)}$.

O projeto foi aprovado pelo Comitê de Ética em Pesquisa do Complexo Hospitalar da Universidade Federal do Ceará e seguiu as normas da Resolução n. ${ }^{\circ}$ 196/96 do Conselho Nacional de Saúde do Ministério da Saúde para pesquisa com seres humanos ${ }^{(14)}$.

Os dados foram organizados em planilha eletrônica (EXCELL) do programa Windows, a análise e interpretação seguiram o tratamento estatístico das informações, sendo discutidos à luz da literatura pertinente.

\section{RESULTADOS}

A Tabela 1 demonstra as freqüências relativas e absolutas dos procedimentos observados. Nos 21 exames observados, o procedimento "verifica se a usuária esvaziou a bexiga" não foi realizado em nenhum atendimento $(100 \%)$, a explicação do exame de prevenção para a paciente foi realizada em 8 (38,1\%), e os procedimentos "orienta a usuária a trocar de roupa atrás da cortina ou biombo, ou no banheiro do consultório, oferecendo-lhe bata" e "cobre a usuária adequadamente com lençol durante o exame físico e/ou ginecológico" foram contemplados pelos enfermeiros em $85,7 \%$ dos atendimentos.

Com relação à classificação dos procedimentos segundo a freqüência relativa de realização nos 21 exames observados, constatou-se que os procedimentos "verifica se a usuária esvaziou a bexiga" e "explica à usuária o que vai ser feito" foram realizados em menos de $70 \%$ dos mesmos, sendo classificados como insatisfatórios. Os procedimentos "orienta a usuária a trocar de roupa atrás da cortina ou biombo, ou no banheiro do consultório, oferecendo-lhe bata" e "cobre a usuária adequadamente com lençol durante o exame físico e/ou ginecológico" tiveram classificação intermediária por terem sido realizados em $85,7 \%$ do total de exames observados, estando, portanto compreendidos na faixa entre $70 \%$ e $90 \%$. Do total dos exames observados, somente $53,4 \%$ foram realizadas todas os procedimentos preconizados pelo Programa de Qualidade-PROQUALI para a coleta de material para o exame de Papanicolaou.

\section{DISCUSSÃO}

A preparação da cliente para o exame compreende os procedimentos relacionados com a interação profissional-cliente, visando o bem-estar e conforto desta. Ao considerar que qualidade está relacionada com a satisfação do cliente, e colocá-lo como foco central do atendimento, verifica-se a importância de realizar essa etapa, pois é basica para que seja estabelecido o relacionamento de confiança entre profissional e cliente ${ }^{(15)}$.

Apesar dos enfermeiros em estudo não terem verificado se, as usuárias esvaziavam a bexiga, sabe-se que esta orientação é importante para promover o conforto da paciente e facilitar o exame, pois a bexiga cheia dificulta o exame dos órgãos pélvicos para o examinador, trazendo incômodo para a cliente ${ }^{(16)}$.

O procedimento "explica à usuária o que vai ser feito" foi contemplado em somente oito atendimentos. Destes, contatou-se que a maioria (seis) foi realizada através de orientação na sala de espera, que também abordou a importância do exame, a técnica, periodicidade de realização e a necessidade de compromisso em retornar para receber o resultado do exame Papanicolaou. Nos demais exames, as explicações foram fornecidas somente às clientes que estavam realizando o exame pela primeira 
vez, o que é questionável pois, o que garante que no primeiro exame as demais usuárias foram esclarecidas?

As relações profissionais-clientes, geralmente, não cumprem o papel educativo que deveriam cumprir, não contribuindo, assim, para melhorar o nível de satisfação das usuárias, o que compromete a qualidade da atenção. Esse fato se dá, primeiramente, pela tradição de ensino dos profissionais de saúde, que privilegia os aspectos técnicos, deixando excluídos, ou incluídos inadequadamente, os aspectos psicossociais e de comunicação $\mathrm{O}^{(9,17)}$. Estudo realizado ${ }^{(8)} \mathrm{em}$ alguns municípios do Estado do Ceará, avaliou o desempenho de 11 enfermeiras na coleta de material para o exame preventivo. Destas, apenas quatro tiveram a preocupação de bem informar à mulher quanto às etapas do exame, constatando a ausência desse cuidado, o que foi considerado como negligência pelas autoras pois, em qualquer atividade, é importante que a cliente saiba a que está sendo submetida, caracterizando-se, inclusive, como um princípio ético do cuidado de Enfermagem ${ }^{(8)}$.

A explicação da técnica deve ocorrer durante o procedimento, encorajando a mulher e conduzindo-a a relaxar, além de fornecer oportunidade para que a mesma formule questionamentos e minimize reações negativas, freqüentemente associadas ao exame ginecológico ${ }^{(16)}$.

$O$ fato de ser um exame que expõe a intimidade feminina e com esta, valores e crenças, muitos são os sentimentos vivenciados pela mulher durante o mesmo. Sentimentos como vergonha, medo e nervosismo são alguns referidos por clientes que são submetidas ao exame $^{(18)}$. Para minimizar tais sentimentos e promover conforto para a paciente, considera-se o respeito à privacidade uma atitude essencial durante a prevenção ${ }^{(19)}$.

Ao orientar a usuária a trocar de roupa atrás da cortina ou biombo, ou no banheiro do consultório, oferecendolhe bata, e ao cobrí-la adequadamente com lençol durante o exame físico e/ou ginecológico, os profissionais agiram numa atitude ética de respeito à privacidade das usuárias.

Entretanto, alguns obstáculos foram observados durante o atendimento à mulher, que dificultaram a manutenção da privacidade da cliente: ausência de banheiro dentro do consultório; portas de consultórios sem fechaduras; e consultórios adaptados com divisórias improvisadas que permitem a escuta do diálogo que se estabelece na consulta, entre o profissional e o cliente. Esses obstáculos poderiam ser minimizados, temporariamente, pelo cuidado de se comunicar em tom de voz mais baixo, porém a infra-estrutura adequada é condição necessária para a qualidade do serviço.

Um serviço de qualidade para o cliente depende da satisfação de alguns requisitos como respeito, compreensão e informação completa e exata. São requisitos que para serem cumpridos não exigem altos custos, mas atitude profissional ética e humanizada ${ }^{(2)}$.
Esses requisitos estão relacionados com o preparo da cliente para o exame, com a relação profissional-cliente, com a manutenção da privacidade e do conforto. Portanto, o preparo da cliente se reflete na qualidade do serviço e na satisfação do cliente.

\section{CONSIDERAÇÕES FINAIS}

O instrumento de melhoria do desempenho (IMD) para prevenção de câncer de colo uterino fornece as diretrizes para um atendimento desejado, tanto para a cliente como para os profissionais. Além disso, fornece parâmetros para avaliar o serviço prestado pela unidade de saúde, estabelecendo um diagnóstico da situação atual deste, denominado "diagnóstico de base".

O diagnóstico de base, ao demonstrar o nível de qualidade do serviço, aponta as deficiências, também referidas como lacunas, encontradas no serviço, impedindo que este alcance padrões de excelência. Nesse contexto, pode-se planejar ações que amenizem ou solucionem as deficiências encontradas, possibilitando melhoria da qualidade desses serviços.

Observou-se, no presente estudo, que apenas em $52,4 \%$, os procedimentos estabelecidos de preparo da mulher para a realização do exame de Papanicolaou foram realizados. O elevado percentual de não realização prejudicou a qualidade do serviço. Vale ressaltar, entretanto, que o respeito à privacidade foi resguardado, merecendo melhoria quanto ao conforto (esvaziar a bexiga) e explicação sobre o procedimento a ser realizado.

Neste contexto, constata-se que o IMD é um instrumento útil para o controle de qualidade, pois oferece os parâmetros práticos e de fácil uso para o monitoramento, supervisão e avaliação, o qual recomendamos para avaliações futuras.

Portanto, ao conhecer e avaliar o preparo de mulheres para o exame de Papanicolaou por enfermeiros, foi possível estabelecer um diagnóstico de base, contendo parâmetros para o planejamento de estratégias de resolução das lacunas apontadas, além de servir como base para posteriores avaliações, contribuindo para melhoria da qualidade dos serviços.

\section{REFERÊNCIAS}

1. Silvia LMV, Formigli VLA. Avaliação em saúde: limites e perspectivas. Cad Saúde Pública Rep Public Health. 1994; 10(1): 80-91.

2. Kols AJ, Sherman JE. Programas de planejamento familiar: melhoria da qualidade. Baltimore: Population Information Program; Johns Hopkins University School of Public Health; 1998. [Population Reports, Serie J, No. 47]

3. Silva AGI. Satisfação do usuário: desvendando as representações sociais sobre a qualidade da assistência de enfermagem. Esc Anna Nery Rev Enferm. 2002; 6(3): 411-23. 
4. Carvalho MLO, Furegato ARF. Exame ginecológico na perspectiva das usuárias de um serviço de saúde. Rev Eletrônjca Enferm [ periódico na internet]. 2001 [citado 2005 Jul 15]; 3(1): [cerca de 12 p.]. Disponível em: http:// www.fen.ufg.br/revista/revista3_1/gineco.html

5. Brenna SMF, Hardy EE, Zeferino LC, Namura I. Conhecimento, atitude e prática do exame de Papanicolaou em mulheres com câncer de colo uterino. Cad Saúde Pública = Rep Public Health. 2001; 17(4): 909-14.

6. Bruce J. Fundamental elements of the quality of care: a simple framework. Stud Fam Plann. 1990; 21(2): 61-91.

7. Brasil. Ministério da Saúde. Instituto Nacional de Câncer. Periodicidade de realização do exame preventivo do câncer do colo do útero: normas e recomendações do INCA. Rev Bras Cancerol. 2002; 48(1): 13-5.

8. Nogueira RA, Moura ERF. Atuação de Enfermeiras nas ações de prevenção do câncer cérvico uterino. Anima. 2004; (6): 37-43.

9. Díaz M, Díaz J. Qualidade de atenção em saúde sexual e reprodutiva: estratégias para mudanças. In: Galvão L, Díaz J, organizadores. Saúde sexual e reprodutiva no Brasil: dilemas e desafios. São Paulo: HUCITEC, Population Council; 1999. p. 210-22.

10. Leopardi MT, Beck CLC, NietscheEA, Gonzáles RMB. Metodologia da Pesquisa na Saúde. Santa Maria: Pallotti; 2001. p. 126- 209.

11. Gil AC. Como elaborar projetos de pesquisa. 4a ed. São
Paulo: Atlas; 2002. p. 41-56.

12. Ceará. Secretaria da Saúde. Metodologia de melhoria da qualidade da atenção à saúde: instrumento de melhoria do desempenho. 2a ed. Fortaleza: SESA-CE; 2005.

13. Polit DF, Beck CT, Hungler BP. Fundamentos de pesquisa em enfermagem: métodos, avaliação e utilização. 5.ed. Porto Alegre: Artmed; 2004.

14. Brasil. Ministério da Saúde. Conselho Nacional de Saúde. Resolução n. 196/96. Diretrizes e normas regulamentadoras de pesquisa envolvendo seres humanos. Diário Oficial da União, Brasília (DF), 16 out. 1996. Seção 1, p.21.082.

15. Ceará. Secretaria de Saúde. Saúde reprodutiva e sexual: um manual para a atenção primária e secundária (nível ambulatorial). Fortaleza: SESA-CE; 2002.

16. Smeltzer SC, Bare BG. Brunner \& Suddarth: tratado de enfermagem médico-cirúrgica. 9. ed. Rio de Janeiro: Guanabara Koogan; 2002. v.3. p. 1137-69.

17. Gamarra CJ, Paz EPA, Griep RH. Conhecimentos, atitudes e prática do exame de Papanicolaou entre mulheres argentinas. Rev Saúde Pública = J Public Health. 2005; 39(2): 270-6.

18. Rodrigues DP, Fernandes AFC, Silva RM. Percepção de algumas mulheres sobre o exame de Papanicolaou. Esc Anna Nery Rev Enferm. 2001; 5(1): 113-8.

19. Barros SMO, Barbieri M, Gerk MA. Exame dos genitais. In: Barros ALBL, et al. Anamnese e exame físico: avaliação diagnóstica de enfermagem no adulto. Porto Alegre: Artmed; 2003. p.179-90.

\section{APENDICE A}

Tabela 1 - Preparo da mulher para a realização do exame de Papanicolaou por enfermeiras do PSF. Paraipaba-CE, 2005

\begin{tabular}{|c|c|c|c|c|c|c|c|c|}
\hline \multirow{2}{*}{ ACOLHIMENTO } & \multicolumn{2}{|c|}{ SIM } & \multicolumn{2}{|c|}{ NÃO } & \multicolumn{2}{|c|}{$\begin{array}{l}\text { NÃO SE } \\
\text { APLICA }\end{array}$} & \multicolumn{2}{|c|}{ TOTAL } \\
\hline & $\mathrm{n}$ & $\%$ & $\mathrm{n}$ & $\%$ & $\%$ & $\mathrm{n}$ & $\mathrm{n}$ & $\%$ \\
\hline Verifica se a usuária esvaziou a bexiga & --- & --- & 21 & 100 & --- & --- & 21 & 100 \\
\hline Explica à usuária o que vai ser feito & 8 & 38,1 & 13 & 61,9 & --- & --- & 21 & 100 \\
\hline $\begin{array}{l}\text { Orienta a usuária a trocar de roupa atrás da cortina ou biombo, ou } \\
\text { no banheiro do consultório, oferecendo-lhe bata, se preciso }\end{array}$ & 18 & 85,7 & 3 & 14,3 & --- & -- & 21 & 100 \\
\hline $\begin{array}{l}\text { Cobre a usuária adequadamente com lençol durante exame físico } \\
\text { e/ou ginecológico, se preciso. }\end{array}$ & 18 & 85,7 & 3 & 14,3 & --- & --- & 21 & 100 \\
\hline
\end{tabular}

